\title{
The effect of arm swing exercise on gait and balance in stroke patients
}

\author{
June-Seok Ma, Hyun-Joo Kim* \\ Department of Physical Therapy, Korea National University of Transportation, ChungJu, Republic of Korea
}

Email address:

devilmaddang@naver.com (A. June-Seok M.), hkim@ut.ac.kr (Hyun-Joo K.)

\section{To cite this article:}

June-Seok Ma, Hyun-Joo Kim. The Effect of Arm Swing Exercise on Gait and Balance in Stroke Patients. American Journal of Clinical and Experimental Medicine. Vol. 2, No. 6, 2014, pp. 151-155. doi: 10.11648/j.ajcem.20140206.16

\begin{abstract}
The aim of this study is investigate the effect of arm swing exercise on balance and gait in stroke patients. Group A ( $n=8)$ : $0.5 \mathrm{~kg}$ strap was applied during arm swing exercise in experimental group for 30 minutes a day, 3 times a week for 4 weeks. Group B $(n=8)$ : Subjects in control group performed neurodevelopmental treatment for 30 minutes a day, 3 times a week for 4 weeks. Subjects were measured for balance and gait on Berg Balance scale, 10meter walking test and Six-minute walk test. There were significant changed of 10Meter Walking Test and 6-Minute Walk Test after experimental group performed the reinforced arm swing exercise of upper extremities and control group was provided with neurodevelopment treatment $(\mathrm{p}<0.05)$. However, there was no statistically significant found difference change of Berg Balance Scale in experimental group $(\mathrm{p}>0.05)$. The application reinforced arm swing exercise can be said to be effective intervention for the gait training in hemiplegic stroke patient.
\end{abstract}

Keywords: Arm Swing, Stroke, Gait

\section{Introduction}

Stroke which is an ischemic or infarct injury of brain results in neurological deficit and contralateral impairments in verbal, cognition, motor and sensory system [1]. Generally, stroke patients are in difficulty for the ability of ADL (Activity of Daily Living) by the combination of motor and sensory impairments which can lead to abnormal gait and balance by disturbing the postural control [2,3].

Hemiplegic stroke patients commonly show the asymmetric weight bearing and use the compensation pattern for the asymmetric posture [4,5]. The ankle strategy to maintain the standing balance is commonly used more than normal adults [6]. Symmetric weight bearing in stroke patients has an effect on the muscle activation of ankle joint through the GRF (Ground Reaction Force) in affected ankle joint [7].

Speed, distance and stability of gait are very important components for the ADL (Activity of Daily Living) in stroke patients [8]. Decrease in gait cycle, speed, standing phase and swing phase of affected side is distinctively shown in stroke patients [9]. Although some patients can recover their gait function, many stroke patients still have not only the disability of decreased gait speed and endurance but also the limitation in independent transfer in home and society [10]. Pohl(2004) reported that the gait speed of stroke patients was a third of normal adults' gait speed after 6 month of onset and stroke patients could perform $40 \%$ of gait distance comparing with normal adults [11]. The improvement and recovery of gait in stroke patients are closely related to the back-to-work and society [12]. Consequentially, the recovery of balance and gait function of stoke patients becomes the important goal of rehabilitation [13].

Normal gait is defined as the movement of extremities which is made by transfer of COG (Center Of Gravity) with effective use of energy [14]. The natural movement which is generated in that situation has an indirect and direct influence and lead to rhythmical gait function with alternative movement of both lower extremities [9]. This overall body balance can reduce the energy consumption in whole body [15].

It is reported that the active movement of affected upper extremities are limited in hemiplegic patients and trunk was not rotated during gait [16]. Furthermore, it is also found by comparing muscle activations of upper extremities that the movement of upper extremities is not related to gait speed and acceleration [17]. Eke-Okoro(1997) reported in his study that natural swing movement of upper extremities has a 
positive effect on the improvement of step length, stride frequency and gait velocity [18]. In addition, powerful swing of upper extremities rather than natural movement is more helpful to weight shift of trunk and gait velocity by increasing the ground reaction force [19]. The movement of upper extremities is also reported to improve the trunk stability and the range of motion on ankle joint [20]. In recent study with normal adults, strengthening exercise with loading on forearm affected the activation of latissimus dorsi muscle, resulting in the increase of the stability in trunk and pelvis [21]. Latissimus dorsi and gluteus maximus are connected by the surface layer of thoracolumbar fascia and this connection can make it possible to transfer the force and rotate the trunk [22]. Shin(2010) reported the effect of symmetric swing exercise of upper extremities on gait speed in hemiplegic patients with stroke [23].

However, there is no study about the effect of strengthening exercise with arm swing upper extremities in stroke patients. Even though many studies have reported the change of gait speed by active and passive movements of upper extremities, there is no study which reported the change of balance and gait. Thus, in this study, the purpose was to investigate the effect of arm swing exercise on balance and gait in stroke patients.

\section{Method}

\subsection{Subjects}

16 patients who diagnosed with stroke by physiatrists were recruited in this study. All subjects were given the information of this study like purpose and procedure and agreed with the participation in this study (consent was given). The inclusion criteria was as following; 1) stroke over 6 months, 2) over 24 points in MMSE-K (Mini-Mental State Examination - Korea version), 3) able to independently sit and stand without any orthosis, 4) able to perform the independent gait for more than six minutes, 5) without subluxation of shoulder, 6) less than second grade of MAS (Modified Ashworth Scale).

In addition, patients who have orthopedic disease, deficit in vision, vestibular system and mental system, and experience of anti-spastic injection were excluded from this study.

\subsection{Experimental Procedure}

All subjects were randomly divided into two groups, experimental and control group. 4-week intervention was provided and subjects performed the following procedure. (1) $0.5 \mathrm{~kg}$ strap was applied during arm swing exercise in experimental group. First stage was that patients put their affected arm out the bobath table in prone position. The extension of affected arm is reinforced with other parts of body fixed. In second stage, the exercise was performed in sitting position. Both feet were on the floor and the maximal extension of affected arm was performed up to $30^{\circ}$ and the maximal flexion was performed less than $30^{\circ}$. Asymmetric pattern was conducted by extending the affected arm during flexion of non-affected arm and flexing the affected arm during extension of non-affected arm. In third stage, the intervention was performed in standing position. Both feet were fixed on the floor with the distance between feet set up by $25 \mathrm{~cm}$. In line with second stage, arm swing exercise of upper extremities was performed. Experimental group performed the exercise for 30 minutes and each stage was conducted for 10 minutes. In each stage, 1-minute resting time was provided after every 2-minute exercise. Interventions were performed 3 times a week for 4 weeks. (2) Subjects in control group performed neurodevelopmental treatment for 30 minutes a day, 3 times a week for 4 weeks.

\subsection{Statistical Analysis}

\section{(1) Berg Balance Scale}

Berg Balance Scale is to measure the balance ability in the elderly or stroke patients and consists of 14 tasks from sit-to-stand to one-leg standing [24]. Each task has 5 scores from 0 to 4 and the full mark 56 points. Intra-rater reliability and inter-rater reliability are $\mathrm{r}=.99$ and $\mathrm{r}=.98$ respectively, showing high validity and reliability [25].

(2) $10 \mathrm{~m}$ walk test

$10 \mathrm{~m}$ walk test is for the measurement of gait speed in patients [26]. $10 \mathrm{~m}$ is marked on the floor and time is measured during $6 \mathrm{~m}$, the middle of $10 \mathrm{~m}$. First $2 \mathrm{~m}$ and last $2 \mathrm{~m}$ are acceleration phase and deceleration phase respectively. Test-retest reliability and inter-rater reliability are $\mathrm{r}=.95$ and $\mathrm{r}=.90$ respectively [27].

(3) Six-minute walk test

6 minute walk test which is a measurement for the gait function and endurance is known to be effective for the measurement of the limited gait by muscle weakness, rigidity, the control impairment and decreased balance in stroke patients. Test-retest reliability is ICC $=0.98$ [28].To investigate the general characteristics of subjects, descriptive statistics and frequency analysis were performed. Kruskal Wallis test was performed to evaluate the changes between before and after the intervention in three groups. Mann-Whitney test was also used for post-hoc comparison. The data were processed using SPSS for Windows Version 20.0 , and a significance level $(\alpha)$ of 0.05 .

\subsection{Statistical Analysis}

SPSS window version 21.0 was used for statistical analysis in this study.

(1) Mann-whitney $U$ test was conducted for the homogeneity test of general characteristics in experimental and control group.

(2) Wilcoxon signed rank some test was used to investigate the change of balance and gait between before and after the intervention in each group.

(3) Mann-whitney U test was also used to investigate the change of balance and gait between groups.

Significance level was .05. 


\section{Result}

\subsection{Homogeneity test of General Characteristics and Various in Experimental and Control Group}

Table 1. General characteristics of subjects (Mean \pm SD)

\begin{tabular}{lllll}
\hline & Group A & Group B & $\mathbf{x}^{2}, \mathbf{U}$ & $\mathbf{p}$ \\
\hline Sex (male / female) & $5 / 3$ & $4 / 4$ & .250 & .617 \\
Stroke side (L / R) & $4 / 4$ & $5 / 3$ & .250 & .617 \\
Age (years) & $57.3 \pm 8.3$ & $54.3 \pm 9.8$ & 22.000 & .292 \\
Weight (kg) & $68.5 \pm 11.1$ & $59.4 \pm 10.1$ & 16.500 & .103 \\
Height (cm) & $164.9 \pm 11.3$ & $160.5 \pm 8.7$ & 27.000 & .598 \\
Onset Time (month) & $8.8 \pm 2.5$ & $8.5 \pm 2.1$ & 31.000 & .915 \\
MMSE-K & $27.75 \pm 2.25$ & $27.8 \pm 2.49$ & 31.500 & .955 \\
MAS & $1.38 \pm 0.52$ & $1.5 \pm 0.53$ & 28.000 & .626 \\
\hline
\end{tabular}

Group $\mathrm{A}=$ Arm swing exercise

Group B = NeuroDevelopmental Treatment

MMSE-K = Mini-Mental State Examination - Korea version

MAS $=$ Modified Ashworth Scale

The general characteristics of experimental and control group were as followings. The ages of both groups were $57.3 \pm 8.3$ and $54.3 \pm 9.8$, and experimental group consists of 5 men and 3 women. Control group consists of 4 men and 4 women. The average scores of MMSE-K (Mini-Mental State Examination-Korea version) were $27.75 \pm 2.25$ in experimental group and $27.8 \pm 2.49$ in control group. The scores of MAS (Modified Ashworth Scale) were $1.38 \pm 0.52$ in experimental group and $1.5 \pm 0.53$ in control group. In statistical analysis, there was no significant difference in all values, thus the homogeneity of both group was confirmed $(\mathrm{p}>0.05)<$ Table $1>$.

In addition, there was no significant difference of gait function and balance ability between both groups, thus the homogeneity of all values between groups before the intervention $(p>0.05)<$ Table $2>$.

Table 2. Pre-homogeneity test for balance and gait

\begin{tabular}{lllll}
\hline & Group A & Group B & U & p \\
\hline BBS & $47.38 \pm 2.88$ & $47.38 \pm 1.69$ & 27.000 & .592 \\
10MWT & $12.51 \pm 1.68$ & $13.01 \pm 2.29$ & 26.000 & .529 \\
6MWT & $152.38 \pm 18.81$ & $125.00 \pm 35.86$ & 14.000 & .059 \\
\hline
\end{tabular}

Mean $\pm \mathrm{SD}=$ Mean \pm Standard Deviation

Group A = Arm swing exercise

Group B = NeuroDevelopmental Treatment

BBS $=$ Berg Balance Scale

$10 \mathrm{MWT}=10$ meter walking test

$6 \mathrm{MWT}=6$-minute Walking test

\subsection{Changes of Gait and Balance Ability}

Wilcoxon signed rank sum test was used to investigate the change of balance after the intervention in both groups and Mann-Whitney $U$ test was also used to investigate the difference of balance ability between groups $<$ Table $3>$.

Table 3. The comparison of balance and gait between experimental and control group General characteristics

\begin{tabular}{|c|c|c|c|c|c|}
\hline & & Group A & Group B & $\mathbf{U}$ & $\mathbf{p}$ \\
\hline \multirow{5}{*}{ BBS } & Pre & $47.38 \pm 2.88$ & $47.38 \pm 1.69$ & & \\
\hline & Post & $48.63 \pm 2.92$ & $49.75 \pm 2.25$ & 15.500 & .077 \\
\hline & Pre-Post & $-1.25 \pm-0.04$ & $-2.37 \pm-0.56$ & & \\
\hline & $\mathrm{z}$ & -1.823 & -2.388 & & \\
\hline & $\mathrm{p}$ & .068 & .017 & & \\
\hline \multirow{3}{*}{ 10MWT } & Pre & $12.51 \pm 1.68$ & $12.85 \pm 2.53$ & & \\
\hline & Post & $8.17 \pm 0.89$ & $10.06 \pm 2.42$ & 11.000 & 0.27 \\
\hline & Pre-Post & $4.34 \pm 0.79$ & $2.79 \pm 0.11$ & & \\
\hline \multirow{7}{*}{ 6MWT } & $\mathrm{z}$ & -2.521 & -2.521 & & \\
\hline & $\mathrm{p}$ & .012 & .012 & & \\
\hline & Pre & $152.38 \pm 18.81$ & $125.00 \pm 35.86$ & & \\
\hline & Post & $188.00 \pm 11.60$ & $145.88 \pm 32.14$ & 10.500 & .023 \\
\hline & Pre-Post & $-35.62 \pm 7.21$ & $-20.88 \pm 3.72$ & & \\
\hline & $\mathrm{z}$ & -2.524 & -2.521 & & \\
\hline & $\mathrm{p}$ & .012 & .012 & & \\
\hline
\end{tabular}

Mean \pm SD $=$ Mean \pm Standard Deviation

Group $\mathrm{A}=$ Arm swing exercise

Group B = NeuroDevelopmental Treatment

BBS $=$ Berg Balance Scale

$10 \mathrm{MWT}=10$ meter walking test

$6 \mathrm{MWT}=6$-minute Walking test

(1) The change of Berg Balance Scale

(1) The change of Berg Balance Scale after the intervention in both groups

Berg Balance Scale score was increased after the intervention from $47.38 \pm 2.88$ to $48.63 \pm 2.92$ in experimental group but not statistically significant. In control group, Berg Balance Scale was significantly increased after the intervention from $47.38 \pm 1.69$ to $49.75 \pm 2.25(\mathrm{p}<0.05)$.

(2) Comparison of BBS scores between groups.

There was no significant difference of Berg Balance Scale between groups $(\mathrm{p}<0.05)$.

(2) Change of 10 Meter walk test

(1) Change of 10Meter Waking Test between before and after the intervention in both groups 
10Meter Waking Test showed significant change after the intervention from $12.51 \pm 1.68 \mathrm{~m} / \mathrm{s}$ to $8.17 \pm 0.89 \mathrm{~m} / \mathrm{s}$ in experimental group. In control group, there was a significant change after the intervention from $12.85 \pm 2.53 \mathrm{~m} / \mathrm{s}$ to $10.06 \pm 2.42 \mathrm{~m} / \mathrm{s}(\mathrm{p}<0.05)$.

(2) Difference of 10Meter Waking Test between groups

There was a significant difference of 10Meter Waking Test between experimental and control group $(p<0.05)$.

(3) Change of 6-Minute Walk Test

(1) Change of 6-Minute Walk Test between before and after the intervention in both groups

6-Minute Walk Test showed statistically significant increase from $152.38 \pm 18.81 \mathrm{~m} / \mathrm{s}$ to $188.00 \pm 11.60 \mathrm{~m} / \mathrm{s}$ after the intervention in experimental group. In control group, there was also significant change after the intervention from $125.00 \pm 35.86 \mathrm{~m} / \mathrm{s}$ to $145.88 \pm 32.14 \mathrm{~m} / \mathrm{s}(\mathrm{p}<0.05)$.

(2) Comparison of 6-Minute Walk Test between experimental and control group

There was a statistically significant difference of 6-Minute Walk Test between experimental and control group $(\mathrm{p}<0.05)$.

\section{Discussion}

Generally, normal gait which is repetitive activity for the body to forward in standing position is a complex movement needing the interaction between joints of lower extremities [29]. If the swing movement of upper extremities is not properly performed, biomechanics and energy are severely affected. In addition, more powerful swing than normal swing is known to increase the ground reaction force and transfer of COG (center of gravity) [30]. Thus, this study was to investigate the effect of reinforced arm swing exercise of upper extremities on the gait and balance in hemiplegic patients with stroke.

In this study, subjects with stroke were divided into two groups and performed the intervention for 30 minutes a day, 3 times a week for 4 weeks. Experimental group was to investigate the change of gait and balance after the reinforced arm swing exercise. The reinforced arm swing exercise was performed in prone, sitting and standing position. Control group was provided with neurodevelopmental treatment.

In the result of this study, there is significant change of 10Merter walk test and 6-Minute Walk Test in experimental group but there is no significant change in Berg Balance Scale. In control group, there are statistically significant changes in all measurement.

$\operatorname{Kim}(2013)$ studied the effects of swing speed of upper extremities during gait on the muscle activation in lower extremities. There was a significant change of muscle activation of latissimus dorsi and gluteus maximus muscle according to the swing speed [21]. There was also significant change of those muscles during natural according to the extinction of loading on upper extremities. In this study, the reinforced arm swing exercise increased the muscle activation of latissimus dorsi and gluteus maximus, and it is thought that arm swing of upper extremities improved the gait distance by increasing gait speed and energy consumption.

Lulic(2008) reported that when patients powerfully swing their upper extremities in intention, the ground reaction force and the movement of COG (Center Of Gravity) were increased [19]. In this study, it is believed that the one-leg standing phase was increased by moving COG (Center Of Gravity) forward and two-leg standing was decreased by increasing the ground reaction force.

$\operatorname{Kim}(2012)$ reported that when intended swing movement of upper extremities increased not only spatiotemporal gait index but also significant improvement of gait speed [16]. In this study, it is also thought for the reinforced arm swing exercise to improve the contraction of latissimus dorsi, resulting in the increase of gait speed and endurance.

However, there was no significant change of Berg Balance Scale after the intervention in this study. Patients used unaffected side more than affected side when performing arm swing exercise. This differentiated the transverse rotation of trunk and as a result, the transverse rotation of pelvis became asymmetric [31,32]. It is thought that the non-significant change is because of that.

There are some limitations in this study. The number of subjects was relatively small then the results were difficult to be generalized to all stroke patients. Furthermore, since patients with subluxation of shoulder and shoulder pain were excluded, there is a difficulty generalizing the result to all stroke patients. Further studies that have larger number of subjects and use the objective measurement tool will be needed in the future.

\section{Conclusion}

In this study, there were significant changes of $10 \mathrm{Meter}$ Walking Test and 6-Minute Waking Test in 16 stroke patients after experimental group performed the reinforced arm swing exercise of upper extremities and control group was provided with neurodevelopment treatment $(p<0.05)$. However, there was no statistically significant change of Berg Balance Scale in experimental group $(\mathrm{p}>0.05)$. Thus, based on this study, it is believed that the reinforced arm swing exercise can be an intervention for the gait training in hemiplegic stroke patients but it is better to provide additional exercise for balance training.

\section{References}

[1] O'Sullivan, SB \& Schmitz, TJ (2007). Physical Rehabilitation: Assessment and Treatment, 5th ed. F.A. Davis Company, Philadelphia, PA, USA.

[2] Walker C, Brouwer BJ, Culham EG. Use of visual feedback in retraining balance following acute stroke. Phys Ther. 2000; 80(9): 886-95.

[3] Smania N, Montagnana B, Faccioli S, Fiaschi A, Aglioti SM. Rehabilitation of somatic sensation and related deficit of motor control in patients with pure sensory stroke. Arch Phys Med Rehabil. 2003; 84(11): 1692-702. 
[4] Geurts AC, de Haart M, van Nes IJ, Duysens J. A review of standing balance recovery from stroke. Gait Posture. 2005; 22(3): 267-81.

[5] Pereira LC, Botelho AC, Martins EF. Relationships between body symmetry during weight-bearing and functional reach among chronic hemiparetic patients. Rev Bras Fisioter. 2010; 14(3): 229-66.

[6] Horvat M, Ray C, Ramsey VK, Miszko T, Keeney R, Blasch BB. Compensatory analysis and strategies for balance in indivisuals with visual impairments. J. Visual Impairment \& Blindness, 2003; 97(11), 695-7033.

[7] Dean CM, Channon EF, Hall JM. Sitting training early after stroke improves sitting ability and quality and carries over to standing up but not to walking: a randomised trial. Aust J Physiother. 2007; 53(2): 97-102.

[8] Dobkin BH, Firestine A, West M, Saremi K, Woods R. Ankle dorsiflexion as an fMRI paradigm to assay motor control for walking during rehabilitation. Neuroimage. 2004; 23(1): 370-81.

[9] Mauritz KH. Gait training in hemiplegia. Eur J Neurol. 2002; 9(1): 23-9.

[10] Chen G, Patten C. Treadmill training with harness support: selection of parameters for individuals with poststroke hemiparesis. J Rehabil Res Dev. 2006 ; 43(4): 485-98.

[11] Pohl PS, Perera S, Duncan PW, Maletsky R, Whitman R, Studenski S. Gains in distance walking in a 3-month follow-up poststroke: what changes? Neurorehabil Neural Repair. 2004; 18(1): 30-6.

[12] Eich HJ, Mach H, Werner C, Hesse S. Aerobic treadmill plus Bobath walking training improves walking in subacute stroke: a randomized controlled trial. Clin Rehabil. 2004; 18(6): 640-51.

[13] Werner C, Von Frankenberg S, Treig T, Konrad M, Hesse S. Treadmill training with partial body weight support and an electromechanical gait trainer for restoration of gait in subacute stroke patients: a randomized crossover study. Stroke. 2002; 33(12): 2895-901.

[14] Ford MP, Wagenaar RC, Newell KM. Arm constraint and walking in healthy adults. Gait Posture. 2007; 26(1): 135-41.

[15] Umberger BR. Effects of suppressing arm swing on kinematics, kinetics, and energetics of human walking. J Biomech. 2008; 41(11): 2575-80

[16] Kim JS, Kwon OH. The effect of arm swing on gait in post-stroke hemiparesis. Department of Physical Therapy. 2012; 7(1): 95-101.

[17] Menz HB, Lord SR, Fitzpatrick RC. Acceleration patterns of the head and pelvis when walking on level and irregular surfaces. Gait Posture. 2003; 18(1): 35-46.

[18] Eke-Okoro ST, Gregoric M, Larsson LE. Alterations in gait resulting from deliberate changes of arm-swing amplitude and phase. Clin Biomech (Bristol, Avon). 1997; 12(7-8): 516-21.
[19] Lulić TJ, Susić A, Kodvanj J. Effects of arm swing on mechanical parameters of human gait. Coll Antropol. 2008; 32(3): 869-73.

[20] Stephenson JL, De Serres SJ, Lamontagne A. The effect of arm movements on the lower limb during gait after a stroke. Gait Posture. 2010; 31(1): 109-15.

[21] Kim TY. The effect of changing gait speed and taking low arm weight on latissimus dorsi and gluteus medius and maximus muscle actiity. Department of Physical Therapy, Graduate school, Inje University, korea, 2013.

[22] Vleeming A, Pool-Goudzwaard AL, Stoeckart R, van Wingerden JP, Snijders CJ. The posterior layer of the thoracolumbar fascia. Its function in load transfer from spine to legs. Spine (Phila Pa 1976). 1995; 20(7): 753-8.

[23] Shin JH, Oh DW. Kim JS. (2010). Effect of nordic walking training on walking function of patients with stroke. Journal of special education \& rehabilitation science. 2013; 49(2): 181-194.

[24] Wee JY, Wong H, Palepu A. Validation of the Berg Balance Scale as a predictor of length of stay and discharge destination in stroke rehabilitation. Arch Phys Med Rehabil. 2003; 84(5): 731-5.

[25] Berg KO, Wood-Dauphinee SL, Williams JI, Maki B. Measuring balance in the elderly: validation of an instrument. Can J Public Health. 1992; 83(2): 7-11.

[26] Deathe AB, Miller WC. The L test of functional mobility: measurement properties of a modified version of the timed "up \& go" test designed for people with lower-limb amputations. Phys Ther. 2005; 85(7): 626-35.

[27] Tilson JK, Sullivan KJ, Cen SY, Rose DK, Koradia CH, Azen SP, Duncan PW. Meaningful gait speed improvement during the first 60 days poststroke: minimal clinically important difference. Phys Ther. 2010; 90(2): 196-208.

[28] Liu J, Drutz C, Kumar R, McVicar L, Weinberger R, Brooks $\mathrm{D}$, Salbach NM. Use of the six-minute walk test poststroke: is there a practice effect? Arch Phys Med Rehabil. 2008; 89(9): 1686-92.

[29] Perry J. Davids JR. Gait analysis: Normal and Pathological function. Journal of Pediatric Orthopaedics. 1992; 12(6): 224-243.

[30] Sigg JA, Ives JC, Gaba BC, Sforzo GA. Effect or arm position on parameters of normal gait in older persons. Clin Kinesio, 1997; 51: 33-36.

[31] Kubo M1, Wagenaar RC, Saltzman E, Holt KG. Biomechanical mechanism for transitions in phase and frequency of arm and leg swing during walking. Biol Cybern. 2004 Aug;91(2): 91-8.

[32] LaFiandra M1, Wagenaar RC, Holt KG, Obusek JP. How do load carriage and walking speed influence trunk coordination and stride parameters? J Biomech. 2003; 36(1): 87-95. 